The inhibitory neurotransmitter glycine modulates macrophage activity by activation of neutral amino acid transporters

Non Peer-reviewed author version

CARMANS, Sofie; HENDRIKS, Jerome; THEWISSEN, Kristof; VAN DEN EYNDEN, Jimmy; STINISSEN, Piet; RIGO, Jean-Michel \& HELLINGS, Niels (2010) The inhibitory neurotransmitter glycine modulates macrophage activity by activation of neutral amino acid transporters. In: JOURNAL OF NEUROSCIENCE RESEARCH, 88(11). p. 2420-2430.

DOI: $10.1002 / j n r .22395$

Handle: http://hdl.handle.net/1942/10704 


\section{The inhibitory neurotransmitter glycine modulates macrophage activity by activation of neutral amino acid transporters}

Sofie Carmans, Jerome J.A. Hendriks, Kristof Thewissen, Jimmy Van den Eynden, Piet Stinissen, JeanMichel Rigo, Niels Hellings *

Hasselt University, Biomedical Research Institute and Transnationale Universiteit Limburg, School of Life Sciences, Diepenbeek, Belgium

Glycine modulates macrophage activity

*Correspondence to Niels Hellings, Biomedical Research Institute, Hasselt University, Agoralaan building C, B-3590 Diepenbeek, Belgium. Tel: + 32112692 68; Fax: + 32112692 99; email: niels.hellings@uhasselt.be

\section{Funded by:}

"Bijzonder onderzoeksfonds" Hasselt University

Nationaal Fonds voor Wetenschappelijk Onderzoek Vlaanderen (FWO)

"Interuniversity Attraction Poles Programme" - Belgian state - Belgian Science Policy 


\begin{abstract}
Glycine, an important inhibitory neurotransmitter in the mammalian central nervous system (CNS), has been shown to modulate peripheral immune cell responses. In that respect, glycine levels are increased in several neuroinflammatory disorders, like amyotrophic lateral sclerosis (ALS) and multiple sclerosis (MS). In this study, we show that glycine modulates macrophage effector functions implicated in CNS inflammation and in other related inflammatory conditions. We demonstrate that glycine does not affect the production of reactive oxygen species, but stimulates myelin phagocytosis and the production of the pro-inflammatory mediators nitric oxide (NO) and tumor necrosis factor (TNF)-a by rat macrophages. These effects of glycine are not mediated by the glycine receptor (GlyR) or by glycine transporters (GlyTs), since neither the GlyR antagonist strychnine, nor the antagonist of GlyT1 (ALX5407) reverse the observed effects. In contrast, 2-aminoisobutyric acid, a substrate of neutral amino acid transporters (NAATs), inhibits the glycine-mediated enhancement of myelin phagocytosis as well as of NO and TNF-a production. In conclusion, our findings demonstrate that glycine modulates macrophage function through activation of NAATs. Glycine may thereby influence immunological processes in inflammatory diseases involving macrophage activation and demyelination, including MS, and related conditions associated with altered glycine levels.
\end{abstract}

\title{
KEYWORDS
}

neuroinflammation $\bullet$ multiple sclerosis $\bullet$ glycine $\bullet$ macrophage $\bullet$ neutral amino acid transporter 


\section{INTRODUCTION}

Glycine is a non-essential amino acid which mediates fast inhibitory neurotransmission within the adult mammalian central nervous system (CNS) (Rajendra et al., 1997). There, glycine exerts its actions by binding to a strychnine-sensitive glycine-gated chloride channel (GlyR), resulting in an increased chloride conductance and an inhibition of excitatory signal transduction (Curtis et al., 1967).

Besides its role as an inhibitory neurotransmitter, glycine also serves functions outside the CNS (Van den Eynden et al., 2008). It has shown to be anti-inflammatory in several extra-neuronal animal models of inflammation (Wheeler et al., 1999). Increasing the blood levels of glycine by dietary administration or intravenous injection reduced the inflammatory responses associated with transplantation (Schemmer et al., 1999), ischemia and reperfusion (Ascher et al., 2001; Zhong et al., 1996) and septic and endotoxic shock (Ikejima et al., 1996; Wheeler et al., 2000). In that respect, a variety of cells involved in inflammation express the GlyR. So far, four a subunits $\left(a_{1}-a_{4}\right)$ and one $\beta$ subunit have been described, that assemble into a pentameric complex with a putative stoichiometry of $2 a: 3 \beta$ or $3 a: 2 \beta$ (Grudzinska et al., 2005). The $\beta$ subunit is required for the clustering of GlyRs, as it binds to the scaffolding protein gephyrin that anchors the GlyR to cytoskeletal elements (Meyer et al., 1995). Froh et al. demonstrated the expression of both a and $\beta$ subunits of the GlyR in rat liver, alveolar and splenic macrophages, with pharmacological properties similar to those of the GlyR in the CNS (Froh et al., 2002). Moreover, glycine has been shown to modulate immune cell functions. In rat alveolar macrophages, glycine inhibited tumor necrosis factor (TNF)-a and superoxide production (Wheeler and Thurman, 1999). Furthermore, glycine reduced the synthesis of TNF-a and interleukin (IL)-1b and enhanced the expression of IL-10 in human monocytes (Spittler et al., 1999). Taken together, these studies indicate that glycine is able to influence immunological processes.

Glycine levels are demonstrated to be increased in various neuronal and non-neuronal inflammatory disorders, like rheumatoid arthritis (Trang et al., 1985), meningitis (Qureshi et al., 1998), amyotrophic lateral sclerosis (ALS) (Iwasaki et al., 1992) and multiple sclerosis (MS) (Barkhatova et al., 1997). Consequently, glycine may influence disease-related inflammatory responses by modulating macrophage function. We aimed to elucidate the effect of glycine on macrophage functions important in neuroinflammatory conditions. 
We demonstrate that glycine does not affect reactive oxygen species (ROS) production, but enhances myelin uptake and production of nitric oxide (NO) and TNF-a by rat peritoneal macrophages. These glycine effects were mediated by neutral amino acid transporter systems (NAATs). This study implicates that glycine may be an important factor in inflammatory diseases involving macrophage activation and demyelination, including MS, as well as in other conditions associated with altered glycine levels.

\section{MATERIALS AND METHODS}

\subsection{Isolation of rat peritoneal macrophages}

Peritoneal macrophages were isolated from 8-10 week old male Wister rats (Harlan, Horst, the Netherlands) by an intra-peritoneal injection of $10 \mathrm{ml}$ of $5 \mathrm{mM}$ EDTA/PBS. After 5 minutes, cells were collected, centrifuged and plated in 24- or 96-well plates at a concentration of $1 \times 10^{6}$ cells $/ \mathrm{ml}$ in MEM culture medium supplemented with $20 \mathrm{mM} \mathrm{L-glutamine,} 50 \mathrm{U} / \mathrm{ml}$ penicillin and $50 \mu \mathrm{g} / \mathrm{ml}$ streptomycin (all Invitrogen, Merelbeke, Belgium) and 10\% heat-inactivated foetal calf serum (FCS; Hyclone, Erembodegem, Belgium). After 2 hours of incubation at $37^{\circ} \mathrm{C}$ and $5 \% \mathrm{CO}_{2}$, non-adherent cells were washed away. The remaining cells were $>95 \%$ macrophages based on flow cytometric analysis of CD11b expression (Hendriks et al., 2008). All procedures were approved by the Ethics Committee for Animals of Hasselt University.

\subsection{RT-PCR amplification of glycine receptor and transporter sequence}

After 24 hours of incubation, mRNA was isolated from rat peritoneal macrophages with the RNeasy Mini Plus Kit (Qiagen, Venlo, the Netherlands), according to the manufacturer's instructions. Subsequently, cDNA was synthesised using the Reverse Transcription system (Promega, Leiden, the Netherlands). CDNA was amplified using a PCR reaction mixture consisting of $25 \mu \mathrm{l}$ of a reaction buffer containing $1 \mathrm{x}$ PCR buffer, $0.20 \mathrm{mM}$ dNTPs, 1 unit of Taq DNA polymerase (all from Roche, Vilvoorde, Belgium) and 1 $\mu \mathrm{M}$ forward and reverse primers (Eurogentec, Seraing, Belgium). See table I for exact primers sequences. Glyceraldehyde-3-phosphate dehydrogenase (GAPDH) was used as housekeeping gene and internal control. The PCR reaction was performed by a BioRad Thermal Cycler (BioRad, Hercules, CA, 
USA). A denaturation step $\left(95^{\circ} \mathrm{C}\right.$ for $5 \mathrm{~min}$ ) was followed by amplification over 40 cycles of repeated denaturation $\left(94^{\circ} \mathrm{C}\right.$ for $\left.20 \mathrm{sec}\right)$, annealing $\left(50-58.7^{\circ} \mathrm{C}\right.$, dependent on the primers, for $\left.20 \mathrm{sec}\right)$ and elongation $\left(72^{\circ} \mathrm{C}\right.$ for $\left.40 \mathrm{sec}\right)$, followed by a $6-\mathrm{min}$ incubation at $72^{\circ} \mathrm{C}$. Reaction products were analysed by $1 \%$ agarose gel electrophoresis.

\subsection{Immunofluorescence of glycine receptor and transporter expression}

Isolated rat peritoneal macrophages were seeded on glass coverslips at a concentration of $3 \times 10^{5}$ cells/coverslip. After 2 hours of incubation at $37^{\circ} \mathrm{C}$ and $5 \% \mathrm{CO}_{2}$, coverslips were washed with PBS to remove unattached cells, fresh culture medium was added and macrophages were incubated for another 24 hours. Cells were fixed by ice cold methanol/acetone $(90 / 10)$ for 10 minutes at $-20^{\circ} \mathrm{C}$. To reduce non-specific binding of antibodies to the macrophages, coverslips were blocked with $3 \%$ goat or donkey serum in PBS for 30 minutes. Subsequently, the following primary antibodies, diluted in PBS, were added during 1 hour at room temperature: mouse mAb4a, which recognises a and $\beta$ GlyR subunits (1:100; Synaptic Systems (SySy), Goettingen, Germany), mouse 3B11 for detection of the anchoring protein gephyrin (1:100; SySy) and goat anti-GlyT1 (1:250, Millipore, Brussels, Belgium). Unbound antibody was removed by washing with PBS and coverslips were further incubated with either goat anti-mouse or donkey anti-goat Alexa 488 secondary antibodies (1:400; Molecular Probes, Invitrogen, the Netherlands), in the dark for 1 hour. Nuclear counterstaining was performed by incubation with 4'-6-Diamidino-2-phenylindole (DAPI; Invitrogen) for 10 minutes in the dark. Finally, coverslips were washed with PBS and mounted onto microscope slides using fluorescent mounting medium (Dako, Glostrup, Denmark). The slides were examined under an Eclipse 80i microscope (Nikon). Negative controls for immunostaining were set up in parallel in the presence of isotype control antibodies.

\subsection{Oxygen radical production}

To assess the effect of glycine on ROS production by macrophages, a dihydrorhodamine 123 (DHR) assay was performed. DHR reacts intracellularly mainly with $\mathrm{H}_{2} \mathrm{O}_{2}$ and becomes oxidized to the fluorescent rhodamine 123 (Rothe et al., 1991).

Peritoneal macrophages were isolated, resuspended and incubated in 24-well plates as described above. Glycine (1 mM) was added for 24,48 or 72 hours. Thereafter, cells were incubated with 100 
$\mathrm{ng} / \mathrm{ml}$ phorbol myristate actetate (PMA; Sigma, Bornem, Belgium) during 15 minutes at $37^{\circ} \mathrm{C}$ in a $5 \%$ $\mathrm{CO}_{2}$ atmosphere. Next, culture medium was removed and $2 \mu \mathrm{M}$ DHR (Sigma) in MEM medium was added for another 15 minutes. Macrophages were washed with ice cold PBS, incubated for 10 minutes on ice with $5 \mathrm{mM}$ EDTA/PBS and detached by vigorous pipetting. The amount of ROS produced was determined by measuring the fluorescence intensity using a FACScalibur flow cytometer (BD Biosciences, Erembodegem, Belgium).

\subsection{Nitric oxide production}

Nitrite $\left(\mathrm{NO}_{2}{ }^{-}\right)$levels, secreted into the supernatant of macrophages cultures, reflect intracellular nitric oxide synthase (iNOS) activity and therefore were taken as a measure of NO production.

Peritoneal macrophages were isolated and seeded in 96 -well plates $\left(1 \times 10^{5}\right.$ cells/well) as described above. Cells were incubated for 24 hours with different concentrations of glycine, the GlyR partial agonists taurine $(1 \mathrm{mM})$ or $\beta$-alanine $(1 \mathrm{mM})$ alone or with $1 \mathrm{mM}$ glycine together with the GlyR antagonist strychnine $(1 \mu \mathrm{M})$, the GlyT1 antagonist ALX5407 (10 nM) or with the neutral amino acid transporter (NAAT) substrate 2-aminoisobutyric acid (AIB; $10 \mathrm{mM}$ ) (All Sigma). Moreover, cells were incubated with $1 \mathrm{mM}$ L-serine with or without $10 \mathrm{mM}$ AIB for 24 hours. Thereafter, cells were stimulated with LPS (10 ng/ml; Calbiochem, VWR international, Leuven, Belgium) and 24 hours later the amount of NO production was assayed with a commercial kit based on the Griess reaction (Griess reagent system; Promega, Leiden, the Netherlands), following the manufacturer's instructions. By using a nitrite standard curve, the concentration of nitrite in the macrophage culture supernatants was calculated.

\subsection{Myelin phagocytosis}

Myelin was prepared from brain tissue of adult Wistar rats by means of sucrose density gradient centrifugation, as previously described by Norton and Poduslo (Norton and Poduslo, 1973). The myelin content was determined using the BCA protein assay kit (Pierce, Thermo Fisher Scientific, Erembodegem, Belgium), which compares a myelin sample to a known BSA standard curve.

Next, myelin was fluorescently labelled according to the method of van der Laan et al (vanderLaan et al., 1996). Shortly, myelin was incubated with the lipophilic dye $1.1^{\prime \prime}$-diotadecyl-3,3,3',3'tetramethylindocarbocyanide perchlorate (DiI; Sigma) at a final concentration of $12.5 \mu \mathrm{g} / \mathrm{ml} \mathrm{DiI}$ on 1 
$\mathrm{mg} / \mathrm{ml}$ myelin during 30 minutes at $37^{\circ} \mathrm{C}$. Excess of DiI was removed by washing with sterile PBS. Labelled myelin was stored in small aliquots at $-20^{\circ} \mathrm{C}$. Rat peritoneal macrophages were isolated and seeded in 24-well plates ( $5 \times 10^{5}$ cells/well) as described above, whereafter $1 \mathrm{mM}$ glycine was added for various time periods. Moreover, macrophages were subjected for 24 hours to the GlyR partial agonists taurine $(1 \mathrm{mM})$ and $\beta$-alanine $(1 \mathrm{mM})$, to various glycine concentrations $(0-3 \mathrm{mM})$ or to $1 \mathrm{mM}$ glycine in the presence of antagonists of the GlyR (strychnine; $1 \mu \mathrm{M}$ ) or GlyT1 (ALX5407; 10 nM). Also, cells were subjected to $1 \mathrm{mM}$ glycine or L-serine alone or in combination with the NAAT substrate AIB $(10 \mathrm{mM})$. Thereafter, a myelin phagocytosis assay was performed as previously described (Hendriks et al., 2008). Briefly, $20 \mu \mathrm{g} /$ well of DiI-labelled myelin was added to the macrophages and cells were incubated for 90 minutes at $37^{\circ} \mathrm{C}$ and $5 \% \mathrm{CO}_{2}$. Thereafter, non-ingested myelin was removed by gently washing the plates with ice cold PBS. For flow cytometric analysis, cells were detached by incubation with $5 \mathrm{mM}$ EDTA/PBS on ice for 10 minutes, followed by vigorous pipetting. The fluorescence intensity, which was used as a measure for binding and uptake of labelled myelin, was determined using a FACScalibur flow cytometer (BD Biosciences).

\subsection{TNF-a production}

Peritoneal macrophages were isolated and seeded in 96 -well plates $\left(1 \times 10^{5}\right.$ cells/well) as described above. Cells were incubated for 24 hours with different concentrations of glycine alone, 1 mM glycine together with the NAAT substrate AIB $(10 \mathrm{mM})$ or with $1 \mathrm{mM}$ L-serine alone or in combination with AIB (10 mM). Next, cells were stimulated with $10 \mathrm{ng} / \mathrm{ml}$ LPS for another 12 hours. Levels of TNF-a, which accumulated in the cell culture supernatant, were measured by using a commercial ELISA kit according to the manufacturer's instructions (eBioscience, Immunosource, Zoersel, Belgium).

\subsection{Macrophage viability}

Peritoneal macrophages were isolated, dispensed into 24- or 96-well plates and exposed to different treatment conditions as described above for the determination of myelin phagocytosis and NO and TNF-a production. After treatment, the viability of the cells used to investigate NO and TNF-a secretion was assessed by a 3-(4, 5-dimethylthiazol-2-yl)-2, 5-diphenyltetrazolium bromide (MTT) assay, according to the method of Mosmann (Mosmann, 1983). Hereto, macrophages were incubated with 0.5 $\mathrm{mg} / \mathrm{ml} \mathrm{MTT} \mathrm{(Sigma)} \mathrm{for} 4$ hours at $37^{\circ} \mathrm{C}$ and $5 \% \mathrm{CO}_{2}$. Unreacted dye was discarded and DMSO/0.1M 
glycine was added to dissolve the produced formazan crystals. The optical density, which is a reflection of cell viability and activity, was measured at $550 \mathrm{~nm}$ using a microplate reader (Biorad). For macrophages used in the myelin phagocytosis assay, cell viability was measured by means of 7-aminoactinomycin $D(7-A A D)$ incorporation. After GlyR agonist/antagonist treatment, macrophages were detached by incubation with $5 \mathrm{mM}$ EDTA/PBS on ice. Next, the cells were incubated with $2.5 \mu \mathrm{g} / \mathrm{ml}$ 7AAD (BD Biosciences) for 20 minutes at $4^{\circ} \mathrm{C}$ in the dark. Samples were analyzed on a FACSCalibur flow cytometer (BD Biosciences) using BD CellQuest software. Apoptotic macrophages were gated based on positive 7-AAD staining.

\subsection{Macrophage proliferation}

To assess the effect of glycine treatment on macrophage proliferation, cells were seeded in 96-well plates $\left(1 \times 10^{5}\right.$ cells/well) as described above. Cells were subjected to $1 \mathrm{mM}$ glycine for 24 hours or to 24 hours pre-treatment with glycine $(1 \mathrm{mM})$ followed by LPS $(10 \mathrm{ng} / \mathrm{ml})$ stimulation for another 24 hours. During the last 8 hours of culture, $1 \mu \mathrm{Ci}\left[{ }^{3} \mathrm{H}\right]$-thymidine (Amersham, Buckinghamshire, UK) was added to each well. Next, cells were harvested with an automatic cell harvester (Pharmacia, Uppsala, Sweden) and incorporation of radioactivity ( $\mathrm{cpm}$ ) was measured in a $\beta$-plate liquid scintillation counter (PerkinElmer Lifesciences, Wellesly, USA).

\subsection{Statistical analysis}

Statistical analysis was performed using the Graphpad Prism4 software package. All results are expressed as mean \pm SEM. Statistical analyses were carried out using the non-parametric KruskalWallis one-way analysis of variance (ANOVA) test with Dunn's post hoc analysis. A significance level of $p<0,05$ was taken to be statistically significant. 


\section{RESULTS}

\subsection{Rat peritoneal macrophages express both the strychnine-sensitive glycine receptor and}

\section{glycine transporter 1}

To elucidate whether glycine modulates macrophage activity, we first evaluated the expression of the GlyR, the GlyR-associated protein gephyrin and the GlyTs on rat peritoneal macrophages. As illustrated in Fig. 1A, rat peritoneal macrophages express mRNAs for the GlyR $\alpha_{1}$ subunit and for gephyrin, an essential protein for the anchoring of the GlyR to cytoskeletal elements, as well as for GlyT1. No mRNA was detected for GlyT2. Moreover, the macrophages showed immunoreactivity for antibodies directed against GlyR subunits (Fig. 1B), gephyrin (Fig. 1C) and GlyT1 (Fig.1D). GlyR and gephyrin staining was mainly located intracellularly (see inset). Negative controls were carried out for each experiment, where cells were labelled with an isotype control antibody and showed no staining (data not shown). It can be concluded that rat peritoneal macrophages express GlyR, gephyrin and GlyT1 both at mRNA and at protein level, suggesting that glycine may modulate macrophage function.

\subsection{Glycine has no effect on ROS production}

Macrophages contribute to the pathology of neuroinflammatory conditions by the production of a variety of pro-inflammatory mediators. ROS produced by macrophages have been shown to cause DNA damage (Lu et al., 2000) and leakage of the blood-brain barrier (Rubanyi, 1988). To investigate whether glycine influences the ROS production by peritoneal rat macrophages, glycine (1 mM) was added to macrophage cultures for 24,48 or 72 hours. Next, ROS production was induced by addition of PMA (100 ng/ml), an activator of protein kinase C. We demonstrate that glycine had no significant effect on the PMA-stimulated ROS production by rat peritoneal macrophages (Fig. 2).

\subsection{Glycine increases NO production in a concentration-dependent manner by activating neutral amino acid transporters}

Another important inflammatory mediator released by macrophages is NO, causing tissue damage and enhancing inflammation. In aqueous solutions, NO reacts with molecular oxygen and accumulates as nitrite $\left(\mathrm{NO}_{2}^{-}\right)$and nitrate $\left(\mathrm{NO}_{3}^{-}\right)$ions. These $\mathrm{NO}$ products can be measured in all kind of fluids and have been used as indicators of NO production both in vitro and in vivo (Koltuksuz et al., 2000). Therefore, 
as a measure of $\mathrm{NO}$ production, we determined the effect of glycine pre-treatment on $\mathrm{NO}_{2}^{-}$levels in culture supernatant of LPS-stimulated rat peritoneal macrophages. The supernatant of unstimulated macrophages did not contain detectable levels of $\mathrm{NO}_{2}{ }^{-}$. Stimulating macrophages with $10 \mathrm{ng} / \mathrm{ml}$ LPS for 24 hours led to an increase in NO production, resulting in $\mathrm{NO}_{2}^{-}$levels of $12.5 \pm 2.5 \mu \mathrm{M}$ (data not shown). The LPS-induced $\mathrm{NO}_{2}^{-}$formation concentration-dependently augmented when macrophage cultures were treated with glycine for 24 hours before LPS addition, as depicted in Fig. 3A. The maximal effect was achieved for $1 \mathrm{mM}$ glycine, which enhanced NO production by $45.3 \pm 10.8 \%$ compared to the LPS-stimulated untreated control $(p<0.01)$. The same was true after interferon- $Y$ (IFN- $\gamma$ ) stimulation $(0.1-100 \mathrm{ng} / \mathrm{ml})$, where $1 \mathrm{mM}$ glycine further upregulated the IFN- $\gamma$ induced increase in NO production (data not shown). The effect on NO production was not due to changes in cell viability or proliferation, as assessed by a 3-(4, 5-dimethylthiazol-2-yl)-2, 5-diphenyltetrazolium bromide (MTT) assay and a $\left[{ }^{3} \mathrm{H}\right]$-thymidine incorporation assay respectively (data not shown).

Next, we investigated if the observed glycine effects on NO production were mediated by the activation of the GlyR. To test this hypothesis, NO production was assessed in the presence of glycine together with strychnine, the prototypic GlyR antagonist which is active at low micromolar concentrations (Ito and Cherubini, 1991). As shown in Fig. 3B, the glycine-evoked increase in NO production was unaffected by $1 \mu \mathrm{M}$ strychnine. Moreover, the GlyR partial agonists taurine $(1 \mathrm{mM})$ and $\beta$-alanine (1 $\mathrm{mM}$ ) did not mimic the effect of glycine, which further supports the idea that the GlyR is not involved in the glycine-evoked enhancement of NO production by rat macrophages.

Since GlyT1 is expressed at the protein level by rat peritoneal macrophages, we next examined whether this GlyT could mediate the effect of glycine. GlyT1 co-transports glycine into the cell in the presence of $\mathrm{Na}^{+}$and $\mathrm{Cl}^{-}$, which in turn can modulate intracellular processes (Eulenburg et al., 2005). To test this, the NO production of LPS-stimulated macrophages pre-treated with $1 \mathrm{mM}$ glycine was assessed in the presence of the specific GlyT1 inhibitor, ALX5407. However, 10 nM ALX5407 did not significantly reverse the glycine-induced increase in NO production (Fig. 4A). Another inhibitor of GlyT1, sarcosine $(60 \mu \mathrm{M})$, was ineffective as well (data not shown). In contrast, in the presence of AIB $(10 \mathrm{mM})$, glycine did not enhance the NO production of macrophages anymore (Fig. 4A). AIB is a synthetic amino acid analogue and substrate for System A and ASC amino acid transporters, which are used to concentrate various amino acids into mammalian cells (Hyde et al., 2003). Glycine is known to be transported by the $\mathrm{Na}^{+}$dependent system $\mathrm{A}, \mathrm{ASC}$ and $\mathrm{L}$ and the $\mathrm{Na}^{+}$-independent system asc 
transporters, the same which carries L-serine into the cell (Hyde et al., 2003; Lecam and Freychet, 1977). Confirming the NAAT involvement, L-serine (1 mM) augmented the NO secretion of macrophages up to $235.3 \pm 11.4 \%$ of LPS-stimulated control $(p<0.001 ;$ Fig. 4B). This increase was reduced to $191.4 \pm 12.1 \%$ by $10 \mathrm{mM}$ AIB $(p<0.05)$. These data strongly indicate that glycine stimulates NO production of rat peritoneal macrophages by activating NAATs in these cells.

\subsection{Glycine increases myelin phagocytosis in a concentration- and time-dependent manner by activating neutral amino acid transporters}

As demyelination is an important aspect associated with neuroinflammation, the binding and internalization of myelin by macrophages was investigated in the presence of glycine. DiI-labelled myelin was added to peritoneal macrophages pre-treated for $6,12,24,48$ or 72 hours with 1 mM glycine. Glycine showed a clear time-dependent stimulation of myelin phagocytosis (Fig. 5A). After 6 hours of glycine treatment, no effect on myelin uptake was observed. However, after 12, 24 and 48 hours of incubation, glycine significantly increased the myelin phagocytosis up to $131.2 \pm 7.3 \%$ $(p<0.01), 134.5 \pm 3.8 \%(p<0.001)$ and $124.1 \pm 5.5 \%(p<0.01)$ compared to untreated macrophages, respectively. After prolonged incubation periods (72 hours), phagocytosis decreased back to control levels. The glycine effect was not only time-dependent, but also concentration-dependent, as shown in Fig. 5B. The level of myelin uptake approached a maximum at $1 \mathrm{mM}$ glycine $(34.5 \pm 3.8 \%$ increase, $\mathrm{p}<0.001$ ), while a higher concentration was less effective. The effect on myelin phagocytosis was not due to any changes in cell viability or proliferation, as assessed by a 7-amino-actinomycin D (7-AAD) incorporation detected by flow cytometric staining and a $\left[{ }^{3} \mathrm{H}\right]$-thymidine incorporation assay respectively (data not shown).

As was the case for NO production, the glycine-induced increase in myelin phagocytosis was not significantly reversed by $1 \mu \mathrm{M}$ strychnine (120.5 $\pm 8.9 \%$ compared to control; Fig. 5C). In addition, neither addition of taurine $(1 \mathrm{mM})$ nor of $\beta$-alanine $(1 \mathrm{mM})$ to the macrophage cultures modified their myelin phagocytosing capacity (Fig. 5C), which further confirmed that glycine promotes myelin phagocytosis in a GlyR-independent way.

Next, we examined the involvement of GlyT1 in the myelin uptake by macrophages. Fig. 6A demonstrates that the specific inhibitor of GlyT1, ALX5407 (10 nM), failed to inhibit the glycine-evoked stimulation of myelin phagocytosis. Similar results were obtained with $60 \mu \mathrm{M}$ sarcosine, another 
inhibitor of GlyT1 (data not shown). These results rule out the possibility that GlyT1 is responsible for the glycine-induced increase in myelin uptake by macrophages.

As shown in Fig. $6 \mathrm{~A}$, incubating macrophages with $1 \mathrm{mM}$ glycine in the presence of $10 \mathrm{mM}$ AIB significantly reversed the glycine-induced increase in myelin phagocytosis from $134.5 \pm 3.8 \%$ to 109.4 $\pm 7.7 \%$ of control $(\mathrm{p}<0.01)$. Moreover, L-serine $(1 \mathrm{mM})$ also increased the myelin uptake by macrophages up to $196.7 \pm 13.7 \%$ of control ( $p<0.001$; Fig. $6 \mathrm{~B}$ ), which was partly counteracted by 10 $\mathrm{mM}$ AIB as well (130.8 $\pm 5.6 \%$ of control). These data demonstrate that glycine enhances the myelin phagocytosing capacity of rat peritoneal macrophages by activating NAATs and may therefore be implicated in myelin removal during neuroinflammation.

\subsection{Glycine concentration-dependently increases the production of TNF-a}

TNF-a is another prototypic product of the inflammatory response of macrophages, which is known to induce cell death and to activate immune cells. Therefore, we studied the effect of glycine treatment on the LPS-induced production of TNF-a by rat peritoneal macrophages. The supernatant of unstimulated macrophages contained very low levels of TNF-a. Stimulating macrophages with $10 \mathrm{ng} / \mathrm{ml}$ LPS for 12 hours, increased the TNF-a production to a concentration of $6.3 \pm 0.4 \mathrm{ng} / \mathrm{ml}$ (data not shown). As depicted in Fig. 7A, pre-treating macrophages for 24 hours with glycine, further concentration-dependently potentiated the LPS-induced TNF-a production. The maximal effect was observed with $1 \mathrm{mM}$ glycine (141.9 $\pm 6.2 \%$ compared to the LPS-stimulated untreated control; $\mathrm{p}<0.001$ ), while a higher concentration was far less potent. Similar results were obtained with $1 \mathrm{mM} \mathrm{L-}$ serine, which augmented the TNF-a production to $152.8 \pm 7.0 \%$ of the LPS-stimulated control cells $(p<0.001$; Fig. 7B). Both $1 \mathrm{mM}$ glycine and L-serine effects were counteracted by the addition of 10 mM AIB. The effect on TNF-a production was not due to changes in cell viability or proliferation, as assessed by a MTT assay and a $\left[{ }^{3} \mathrm{H}\right]$-thymidine incorporation assay respectively (data not shown). These results demonstrate that glycine enhances the LPS-induced production of TNF-a by peritoneal macrophages, most likely by triggering NAATs. 


\section{DISCUSSION}

Glycine, a well-known inhibitory neurotransmitter in the mammalian spinal cord and brain stem (Curtis et al., 1967; Rajendra et al., 1997), has been shown to have beneficial effects in various animal models of peripheral inflammation. In that respect, different immune cells seem to be modulated by glycine (Gundersen et al., 2005; Wheeler et al., 1999). In the current study, we demonstrate that glycine does not affect ROS production, but increases the production of the pro-inflammatory mediators NO and TNF-a, and also enhances myelin phagocytosis by rat peritoneal macrophages.

To our knowledge, we are the first to demonstrate that glycine modulates macrophage function independently of the GlyR or GlyTs, but rather by the activation of NAATs, transporter systems used by mammalian cells to concentrate amino acids in the cell. In line with our finding, Schilling et al. reported that glycine-evoked currents in mouse microglia are mediated by the activation of NAATs (Schilling and Eder, 2004). We show that in the presence of AIB, a substrate for System A and ASC amino acid transporters (Hyde et al., 2003), glycine no longer increased the production of TNF-a and of NO nor the myelin uptake by macrophages. Moreover, L-serine reproduced the effect of glycine, which in turn could be counteracted by AIB. However, AIB was not always able to fully reverse the effect of glycine and L-serine. This might be explained by the fact that glycine and L-serine are known to be transported by the $\mathrm{Na}^{+}$dependent System $\mathrm{A}, \mathrm{ASC}$ and $\mathrm{L}$ and by the $\mathrm{Na}^{+}$-independent system asc transporters (Hyde et al., 2003; Lecam and Freychet, 1977), and thus by additional NAATs then those activated by AIB.

So far, it is unclear how glycine transport by NAATs leads to an altered macrophage activity. Glycine may increase the intracellular $\mathrm{Na}^{+}$concentration, as activation of system A transporters couples amino acid influx to $\mathrm{Na}^{+}$influx (Mackenzie and Erickson, 2004; Schilling and Eder, 2004). This leads to a depolarization of the cell membrane, which in turn will augment the $\mathrm{Ca}^{2+}$ influx by voltage-operated $\mathrm{Ca}^{2+}$ channels, as shown for Kupffer cells (Hijioka et al., 1992). The resulting increase in $\left[\mathrm{Ca}^{2+}\right]_{\mathrm{i}}$ could in turn activate $\mathrm{Ca}^{2+}$-dependent pathways, which are involved in the induction of several inflammatory responses by macrophages, like phagocytosis and the production of NO and TNF-a (Cohen et al., 2006; Hotchkiss et al., 1997; Watanabe et al., 1996). TNF-a and NO are involved in various aspects of CNS inflammatory responses. Both increase axonal vulnerability, as they induce damage to myelin and oligodendrocytes (Selmaj and Raine, 1988; Slaets et al., 2008; Touil et al., 2001). Moreover, 
stimulation of myelin uptake facilitates demyelination and axonal degeneration (Vercellino et al., 2009). However, NO and TNF-a can be beneficial as well, by inhibiting leukocyte infiltration and promoting the proliferation of oligodendrocyte progenitors and remyelination, respectively (Arnett et al., 2001; Kubes et al., 1991). Therefore, by enhancing myelin uptake and NO and TNF-a production by macrophages, glycine may modulate CNS inflammatory insults.

In other macrophage subtypes, such as rat alveolar macrophages and Kupffer cells, the TNF-a and superoxide production was reduced after glycine treatment (Ikejima and Thurman, 1995; Wheeler and Thurman, 1999). Since molecular evidence suggests the existence of GlyRs on liver, alveolar and splenic macrophages (Froh et al., 2002), the reported anti-inflammatory effects have up to now mainly been explained by GlyR activation, induced by glycine and antagonized by strychnine treatment (Froh et al., 2002; Ikejima et al., 1997; Wheeler and Thurman, 1999). In our experimental set-up, we did not observe a reversal of glycine-evoked effects by strychnine. In addition, the GlyR partial agonists taurine and $\beta$-alanine failed to mimic the glycine-induced effects. The observed glycine effects are neither mediated by GlyTs, although GlyT1 is expressed by rat peritoneal macrophages. To our knowledge, we are the first to demonstrate the expression of GlyT1 on rat peritoneal macrophages. Until now, GlyT1 was demonstrated on neurons, astrocytes and oligodendrocyte progenitors (Adams et al., 1995) and in several peripheral tissues (Jursky and Nelson, 1996). In addition, we show that rat peritoneal macrophages express the GlyR $a_{1}$ subunit, but not the $\alpha_{2}-\alpha_{4}$ or $\beta$ subunits, which confirms GlyR a-subunit heterogeneity within the macrophage population as already demonstrated by Froh et al. (Froh et al., 2002). Finally, in peritoneal macrophages, the GlyR seems to be mainly located intracellularly and hence their expression of strychnine-sensitive functional GlyRs remains elusive.

Glycine is thus able to influence macrophages by different mechanisms, namely GlyR-independently and NAAT-dependently as reported here, versus GlyR-dependently, which might explain the discrepancies of glycine effects between our study and the ones previously reported. It is yet unclear if this expression is specific for macrophage subtypes or if it is a result of the macrophage phenotype, activation or functional status, of which the last has already been reported for other ion channels in peripheral tissue macrophages and brain macrophages (Eder, 1998). One might speculate that the GlyR-independent effects mediated by NAATs lead to an augmented $\left[\mathrm{Ca}^{2+}\right]_{\mathrm{i}}$, while those mediated by GlyRs would oppose the increase in $\left[\mathrm{Ca}^{2+}\right]_{i}$, resulting in respectively pro- and anti-inflammatory macrophage effector functions. We therefore hypothesize that in various conditions with altered glycine 
levels, glycine modulates macrophage activity in different manners, which in turn dictates a proinflammatory GlyR-independent versus an anti-inflammatory GlyR-dependent outcome.

The GlyR- and GlyT-independency but NAAT-dependency of glycine-mediated effects has also been shown in mouse microglia (Schilling and Eder, 2004). We made use of peritoneal macrophages which, comparable to CNS infiltrating macrophages, are derived from monocytes in the circulation (Vanfurth and Cohn, 1968). Glycine levels are known to be increased in several neuroinflammatory disorders, like ALS (Iwasaki et al., 1992) and MS (Barkhatova et al., 1997). Moreover, glycine concentrations needed in our study to evoke glycine-mediated enhancement of macrophage activity ( $\geq 100 \mu \mathrm{M})$ are within the range detected in inflammatory conditions ( $\pm 250 \mu \mathrm{M})$ (Wesseldijk et al., 2008). Consequently, our findings indicate that in CNS diseases with altered glycine concentrations in the CNS as well as in the periphery, glycine may affect the function of resident microglia and invading macrophages, as well as peripheral macrophages and blood monocytes, by a similar mechanism.

Overall, we demonstrate that glycine is immunomodulatory as it increases the production of the proinflammatory mediators NO and TNF-a and enhances the phagocytosis of myelin by macrophages. These effects are not mediated by the GlyR or GlyTs, but implicate the activation of NAATs. These findings gain new insight into the role of glycine in CNS diseases and inflammatory disorders involving macrophage activation and demyelination, including MS and related conditions with altered glycine levels.

\section{ACKNOWLEDGEMENTS}

We would like to thank W. Leyssens for technical assistance and S. Sahebali for assistance with the PCR and immunofluorescent stainings. S.C. holds a fellowship from the 'Bijzonder Onderzoeksfonds' Hasselt University. 


\section{REFERENCES}

Adams RH, Sato K, Shimada S, Tohyama M, Puschel AW, Betz H. 1995. Gene Structure and Glial Expression of the Glycine Transporter Glyt1 in Embryonic and Adult Rodents. J Neurosci 15:2524-2532. Arnett HA, Mason J, Marino M, Suzuki K, Matsushima GK, Ting JPY. 2001. TNF alpha promotes proliferation of oligodendrocyte progenitors and remyelination. Nat Neurosci 4:1116-1122.

Ascher E, Hanson JN, Cheng W, Hingorani A, Scheinman M. 2001. Glycine preserves function and decreases necrosis in skeletal muscle undergoing ischemia and reperfusion injury. Surgery 129:231235.

Barkhatova VP, Zavalishin IA, Askarova LS, Shavratsky VK, Demina EG. 1997. Neurotransmitters changes in multiple sclerosis. Zhurnal Nevropatologii I Psikhiatrii Imeni S S Korsakova 97:7-10.

Cohen G, Makranz C, Spira M, Kodama T, Reichert F, Rotshenker S. 2006. Non-PKC DAG/phorbol-ester receptor(s) inhibit complement receptor-3 and nPKC inhibit scavenger receptor-AI/II mediated myelin phagocytosis but CPKC, PI3K, and PLC gamma activate myelin phagocytosis by both. Glia 53:538-550.

Curtis DR, Hosli L, Johnston GA. 1967. Inhibition of Spinal Neurones by Glycine. Nature 215:15021503.

Eder C. 1998. Ion channels in microglia (brain macrophages). Am J Phys Cell Phys 44:C327-C342.

Eulenburg V, Armsen W, Betz H, Gomeza J. 2005. Glycine transporters: essential regulators of neurotransmission. Trends Biochem Sci 30:325-333.

Froh M, Thurman RG, Wheeler MD. 2002. Molecular evidence for a glycine-gated chloride channel in macrophages and leukocytes. Am J Physiol Gastrointest Liver Physiol 283:G856-G863.

Grudzinska J, Schemm R, Haeger S, Nicke A, Schmalzing G, Betz H, Laube B. 2005. The beta subunit determines the ligand binding properties of synaptic glycine receptors. Neuron 45:727-739.

Gundersen RY, Vaagenes P, Breivik T, Fonnum F, Opstad PK. 2005. Glycine - an important neurotransmitter and cytoprotective agent. Acta Anaesthesiol Scand 49:1108-1116.

Hendriks JJA, Slaets H, Carmans S, de Vries HE, Dijkstra CD, Stinissen P, Hellings N. 2008. Leukemia inhibitory factor modulates production of inflammatory mediators and myelin phagocytosis by macrophages. J Neuroimmunol 204:52-57. 
Hijioka T, Rosenberg RL, Lemasters JJ, Thurman RG. 1992. Kupffer Cells Contain Voltage-Dependent Calcium Channels. Mol Pharmacol 41:435-440.

Hotchkiss RS, Bowling WM, Karl IE, Osborne DF, Flye MW. 1997. Calcium antagonists inhibit oxidative burst and nitrite formation in lipopolysaccharide-stimulated rat peritoneal macrophages. Shock 8:170178.

Hyde R, Taylor PM, Hundal HS. 2003. Amino acid transporters: roles in amino acid sensing and signalling in animal cells. Biochem J 373:1-18.

Ikejima K, Iimuro Y, Forman DT, Thurman RG. 1996. A diet containing glycine improves survival in endotoxin shock in the rat. Am J Physiol Gastrointest Liver Physiol 34:G97-G103.

Ikejima K, Qu W, Stachlewitz RF, Thurman RG. 1997. Kupffer cells contain a glycine-gated chloride channel. Am J Physiol Gastrointest Liver Physiol 35:G1581-G1586.

Ikejima K, Thurman RG. 1995. Glycine Prevents Elevation in Intracellular Ca2+ in Kupffer Cells by Endotoxin (Lps) Via Actions on A Chloride Channel. Hepatology 22:803.

Ito S, Cherubini E. 1991. Strychnine-Sensitive Glycine Responses of Neonatal Rat HippocampalNeurons. Journal of Physiology-London 440:67-83.

Iwasaki Y, Ikeda K, Kinoshita M. 1992. Plasma Amino-Acid Levels in Patients with Amyotrophic-LateralSclerosis. J Neurol Sci 107:219-222.

Jursky F, Nelson N. 1996. Developmental expression of the glycine transporters GLYT1 and GLYT2 in mouse brain. J Neurochem 67:336-344.

Koltuksuz U, Irmak MK, Karaman A, Uz E, Var A, Ozyurt H, Akyol O. 2000. Testicular nitric oxide levels after unilateral testicular torsion/detorsion in rats pretreated with caffeic acid phenethyl ester. Urol Res $28: 360-363$.

Kubes P, Suzuki M, Granger DN. 1991. Nitric-Oxide - An Endogenous Modulator of Leukocyte Adhesion. Proc Natl Acad Sci U S A 88:4651-4655.

Lecam A, Freychet P. 1977. Neutral Amino-Acid Transport - Characterization of A and L-Systems in Isolated Rat Hepatocytes. J Biol Chem 252:148-156.

Lu FM, Selak M, O'Connor J, Croul S, Lorenzana C, Butunoi C, Kalman B. 2000. Oxidative damage to mitochondrial DNA and activity of mitochondrial enzymes in chronic active lesions of multiple sclerosis. J Neurol Sci 177:95-103. 
Mackenzie B, Erickson JD. 2004. Sodium-coupled neutral amino acid (System N/A) transporters of the SLC38 gene family. Pflugers Arch 447:784-795.

Meyer G, Kirsch J, Betz H, Langosch D. 1995. Identification of A Gephyrin Binding Motif on the Glycine Receptor-Beta Subunit. Neuron 15:563-572.

Mosmann T. 1983. Rapid Colorimetric Assay for Cellular Growth and Survival - Application to Proliferation and Cyto-Toxicity Assays. J Immunol Methods 65:55-63.

Norton WT, Poduslo SE. 1973. Myelination in Rat-Brain - Method of Myelin Isolation. J Neurochem 21:749-757.

Qureshi GA, Baig SM, Bednar I, Halawa A, Parvez SH. 1998. The neurochemical markers in cerebrospinal fluid to differentiate between aseptic and tuberculous meningitis. Neurochem Int 32:197203.

Rajendra S, Lynch JW, Schofield PR. 1997. The glycine receptor. Pharmacol Ther 73:121-146.

Rothe G, Emmendorffer A, Oser A, Roesler J, Valet G. 1991. Flow Cytometric Measurement of the Respiratory Burst Activity of Phagocytes Using Dihydrorhodamine 123. J Immunol Methods 138:133135.

Rubanyi GM. 1988. Vascular Effects of Oxygen-Derived Free-Radicals. Free Radic Biol Med 4:107-120.

Schemmer P, Bradford BU, Rose ML, Bunzendahl H, Raleigh JA, Lemasters JJ, Thurman RG. 1999. Intravenous glycine improves survival in rat liver transplantation. Am J Physiol Gastrointest Liver Physiol 276:G924-G932.

Schilling T, Eder C. 2004. A novel physiological mechanism of glycine-induced immunomodulation: $\mathrm{Na}+$-coupled amino acid transporter currents in cultured brain macrophages. J Physiol (Lond) 559:3540.

Selmaj KW, Raine CS. 1988. Tumor Necrosis Factor Mediates Myelin and Oligodendrocyte Damage Invitro. Ann Neurol 23:339-346.

Slaets H, Dumont D, Vanderlocht J, Noben JP, Leprince P, Robben J, Hendriks J, Stinissen P, Hellings N. 2008. Leukemia inhibitory factor induces an antiapoptotic response in oligodendrocytes through Aktphosphorylation and up-regulation of 14-3-3. Proteomics 8:1237-1247.

Spittler A, Reissner CM, Oehler R, Gornikiewicz A, Gruenberger T, Manhart N, Brodowicz T, Mittlboeck M, Boltz-Nitulescu G, Roth E. 1999. Immunomodulatory effects of glycine on LPS-treated monocytes: reduced TNF-alpha production and accelerated IL-10 expression. FASEB J 13:563-571. 
Touil T, Deloire-Grassin MSA, Vital C, Petry KG, Brochet B. 2001. In vivo damage of CNS myelin and axons induced by peroxynitrite. Neuroreport 12:3637-3644.

Trang LE, Furst P, Odeback AC, Lovgren O. 1985. Plasma Amino-Acids in Rheumatoid-Arthritis. Scand J Rheumatol 14:393-402.

Van den Eynden J, Sahebali S, Horwood N, Carmans S, Brône B, Hellings N, Steels P, Harvey RJ, Rigo JM. 2008. Glycine and glycine receptor signalling in non-neuronal cells. Front Mol Neurosci 2.

vanderLaan LJW, Ruuls SR, Weber KS, Lodder IJ, Dopp EA, Dijkstra CD. 1996. Macrophage phagocytosis of myelin in vitro determined by flow cytometry: Phagocytosis is mediated by CR3 and induces production of tumor necrosis factor-alpha and nitric oxide. J Neuroimmunol 70:145-152.

Vanfurth R, Cohn ZA. 1968. Origin and Kinetics of Mononuclear Phagocytes. J Exp Med 128:415-435.

Vercellino M, Masera S, Lorenzatti M, Condello C, Merola A, Mattioda A, Tribolo A, Capello E, Mancardi GL, Mutani R, Giordana MT, Cavalla P. 2009. Demyelination, Inflammation, and Neurodegeneration in Multiple Sclerosis Deep Gray Matter. J Neuropathol Exp Neurol 68:489-502.

Watanabe N, Suzuki J, Kobayashi Y. 1996. Role of calcium in tumor necrosis factor-alpha production by activated macrophages. J Biochem (Tokyo) 120:1190-1195.

Wesseldijk F, Fekkes D, Huygen FJPM, Heide-Mulder M, Zijlstra FJ. 2008. Increased plasma glutamate, glycine, and arginine levels in complex regional pain syndrome type 1. Acta Anaesthesiol Scand 52:688-694.

Wheeler MD, Ikejema K, Enomoto N, Stacklewitz RF, Seabra V, Zhong Z, Yin M, Schemmer P, Rose ML, Rusyn I, Bradford B, Thurman RG. 1999. Glycine: a new anti-inflammatory immunonutrient. Cell Mol Life Sci 56:843-856.

Wheeler MD, Rose ML, Yamashima S, Enomoto N, Seabra V, Madren J, Thurman RG. 2000. Dietary glycine blunts lung inflammatory cell influx following acute endotoxin. Am J Physiol Lung Cell Mol Physiol 279:L390-L398.

Wheeler MD, Thurman RG. 1999. Production of superoxide and TNF-alpha from alveolar macrophages is blunted by glycine. Am J Physiol 277:L952-L959.

Zhong Z, Jones S, Thurman RG. 1996. Glycine minimizes reperfusion injury in a low-flow, reflow liver perfusion model in the rat. Am J Physiol Gastrointest Liver Physiol 33:G332-G338. 


\section{FIGURES}
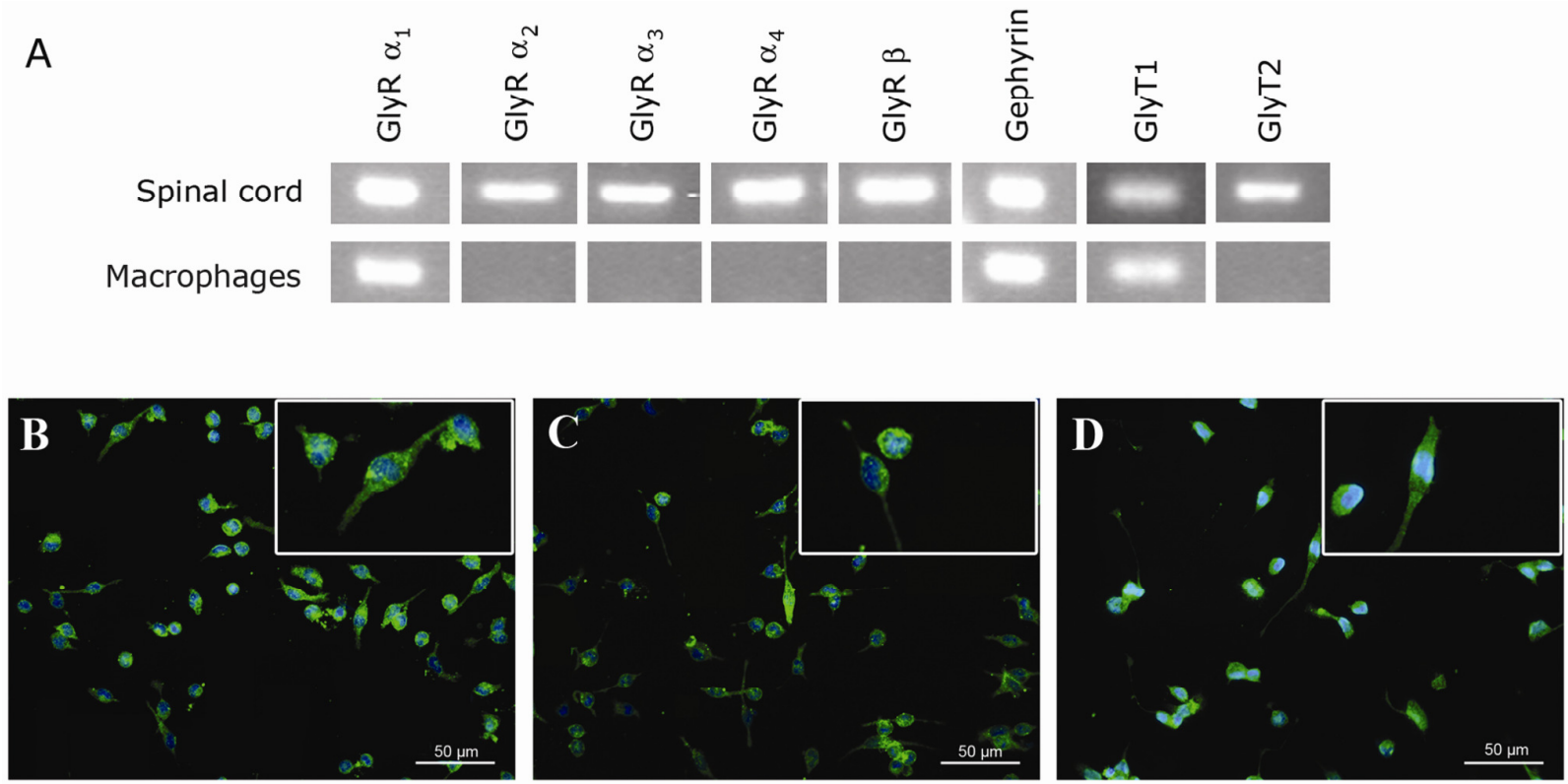

Fig. 1. mRNA and immunocytochemical evidences for glycine receptor, its associated protein gephyrin and glycine transporter 1 in peritoneal rat macrophages. (A) RT-PCR analysis demonstrates the expression of GlyR $\alpha_{1}$ subunit, gephyrin and GlyT1 mRNA in rat peritoneal macrophages. Rat spinal cord was used as a positive control, expressing all GlyR subunits and GlyTs. (B-D) Fluorescent immunocytochemistry using secondary Alexa-488 (green) antibody staining showed the presence of the glycine receptor (GlyR) (B), the GlyR-associated protein gephyrin (C), as well as the glycine transporter GlyT1 (D) on isolated peritoneal rat macrophages. The immunolabelling for GlyR and gephyrin showed intracellular localisation (see in inset). Nuclear counterstaining was performed by DAPI (blue). 


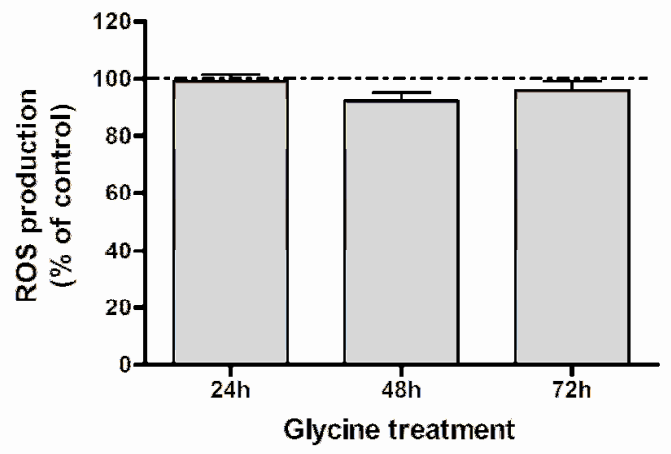

Fig. 2. Glycine does not affect the PMA-induced production of ROS by macrophages. Rat peritoneal macrophages were incubated with glycine for 24,48 or $72 \mathrm{~h}$, followed by 15 min stimulation with $100 \mathrm{ng} / \mathrm{ml}$ PMA and subsequent staining for 15 min with $2 \mu \mathrm{M}$ DHR-123. Glycine (1 mM) does not affect ROS production by PMA-stimulated macrophages at any time point. ROS production is plotted as mean percentage of ROS production by PMA-stimulated untreated control cells on the corresponding day \pm SEM. Data are derived from 6 independent experiments with quadruplet measurements for each experiment. 

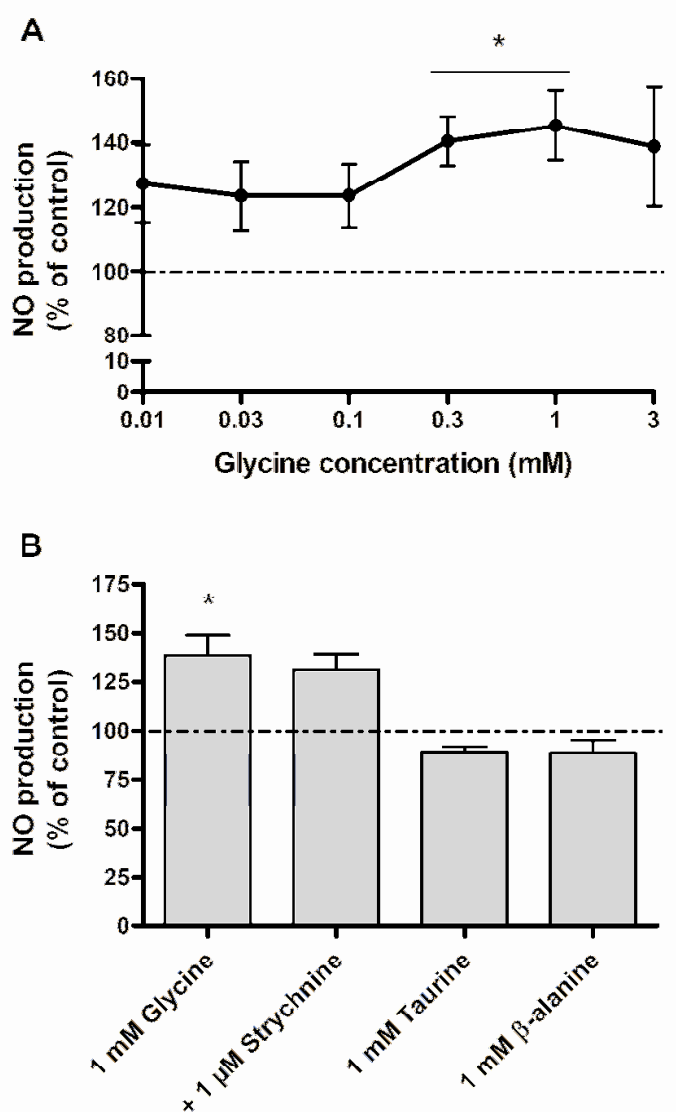

Fig. 3. Glycine stimulates LPS-induced NO production in a GlyR-independent way. (A) Pretreating peritoneal macrophages for $24 \mathrm{~h}$ with glycine, followed by LPS $(10 \mathrm{ng} / \mathrm{ml})$ stimulation for another $24 \mathrm{~h}$, concentration-dependently increased the NO production. (B) Rat macrophages were pretreated for $24 \mathrm{~h}$ with the GlyR agonists $(1 \mathrm{mM})$ glycine, taurine or $\beta$-alanine alone or glycine together with the GlyR antagonist strychnine $(1 \mu \mathrm{M})$. Afterwards, $10 \mathrm{ng} / \mathrm{ml}$ LPS was added for $24 \mathrm{~h}$. Strychnine was not able to reverse the glycine-induced increase in NO production. The GlyR partial agonists taurine and $\beta$-alanine had no effect on NO production, which indicates a GlyR-independent mechanism of action. Data shown are plotted as mean percentage of NO production by LPS-stimulated untreated macrophages \pm SEM. $100 \%$ corresponds with a mean of $12.5 \pm 2.5 \mu \mathrm{M}$ NO. Data are derived from at least 4 independent experiments with triplicate measurements per experiment. $* p<0.01$ compared to LPS-stimulated untreated cells (by Kruskal-Wallis test and Dunn's post hoc analysis). 

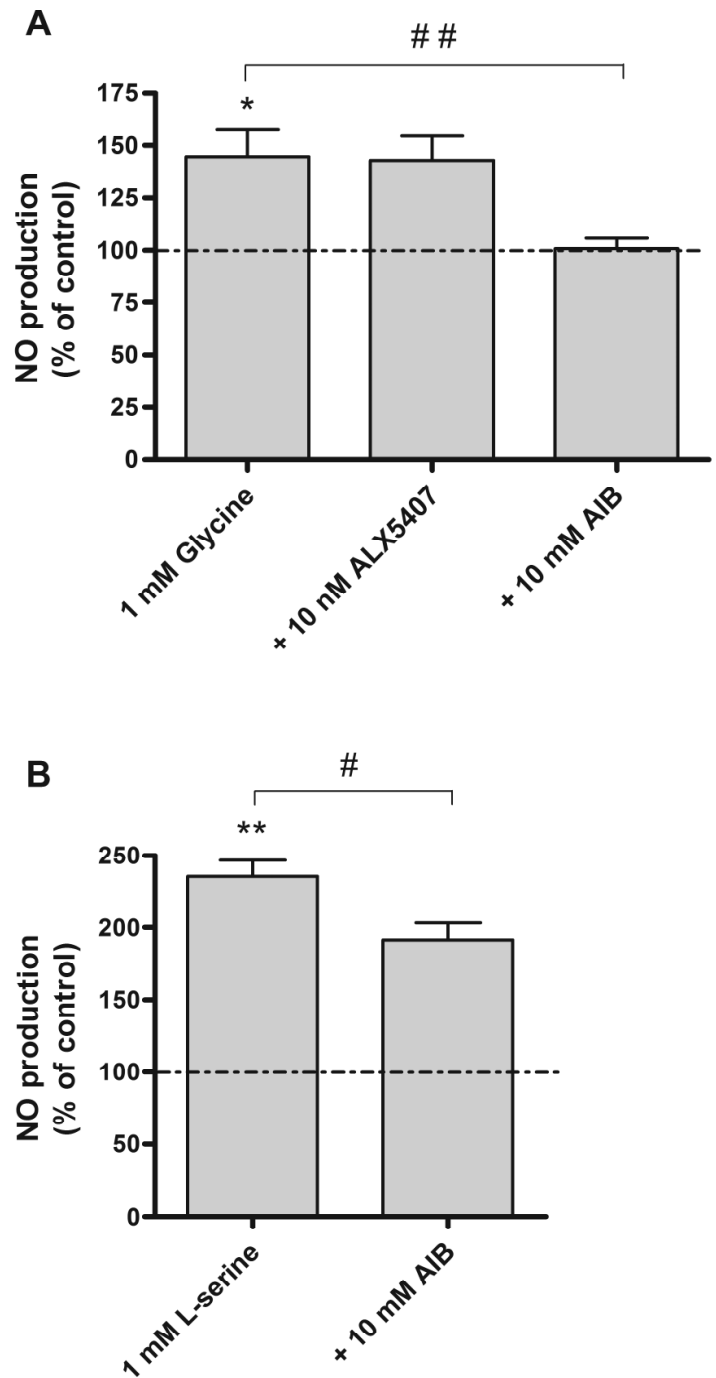

Fig. 4. Glycine modulates NO production by macrophages by the activation of neutral amino acid transporters. (A) Co-incubation of $1 \mathrm{mM}$ glycine with the GlyT1 inhibitor ALX5407 (10 nM) did not abolish the glycine-enhanced NO production. In contrast, $10 \mathrm{mM}$ 2-aminoisobutyric acid (AIB), a substrate of neutral amino acid transporters (NAATs), reversed the glycine-induced increase in NO production. (B) Treating macrophages with L-serine (1 mM) also enhanced the NO production by LPSstimulated macrophages. This was counteracted, at least partially, by AIB. Data shown are plotted as mean percentage of NO production by LPS-stimulated untreated macrophages \pm SEM. Data are derived from at least 4 independent experiments with triplicate measurements per experiment. $* p<0.01 ; * *$ $\mathrm{p}<0.001$ compared to LPS-stimulated untreated macrophages. \# $\mathrm{p}<0.05 ; \# \# \mathrm{p}<0.001$ compared to 1 
mM L-serine/glycine treated LPS-stimulated macrophages (by Kruskal-Wallis test and Dunn's post hoc analysis).

A

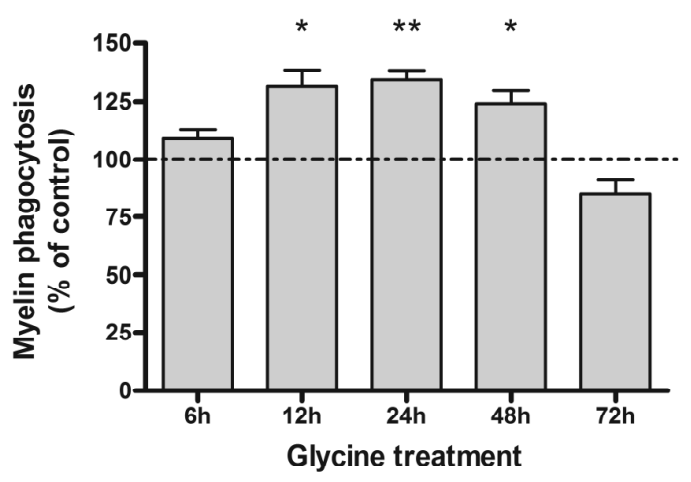

C

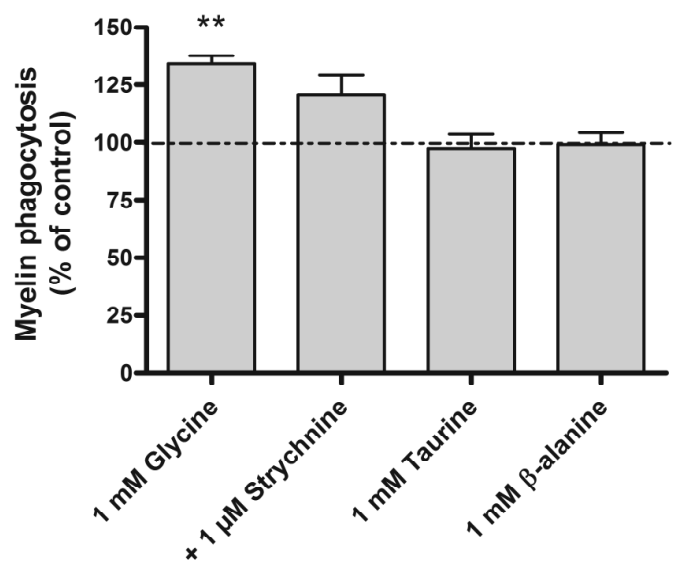

B

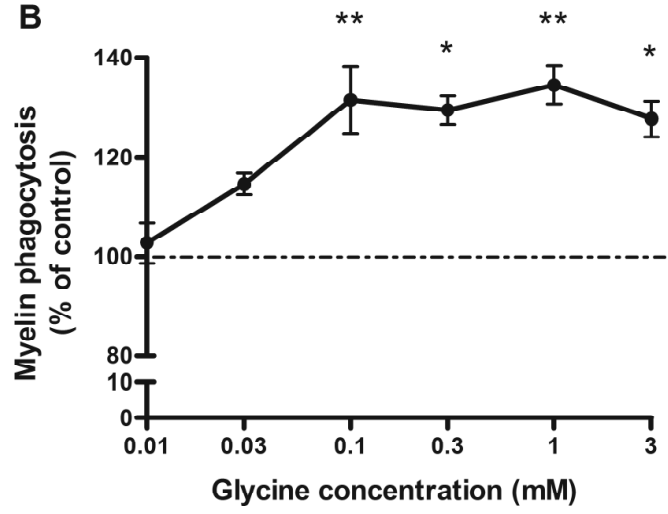

Fig. 5. Glycine time- and concentration-dependently stimulates myelin uptake by

macrophages. The myelin phagocytosing capacity of macrophages was determined by adding DiIlabelled myelin to glycine-treated macrophage cultures for $90 \mathrm{~min}$. (A) The myelin uptake by rat macrophages was significantly increased by $1 \mathrm{mM}$ glycine after 12,24 and $48 \mathrm{~h}$ of treatment. (B) $24 \mathrm{~h}$ treatment with glycine resulted in a concentration-dependent stimulation of myelin uptake by macrophages and was maximal at a concentration of $1 \mathrm{mM}$ glycine. (C) Neither co-incubation of $1 \mathrm{mM}$ glycine with the GlyR antagonist strychnine $(1 \mu \mathrm{M})$, nor treatment with the partial agonists taurine (1 $\mathrm{mM}$ ) or $\beta$-alanine $(1 \mathrm{mM})$ modified the myelin uptake of macrophages. Data shown are plotted as mean percentage of myelin phagocytosis by untreated macrophages \pm SEM as measured by flow cytometry. Data shown are derived from at least 4 independent experiments with triplicate measurements in each 
experiment. * $\mathrm{p}<0.01$; ** $\mathrm{p}<0.001$ compared to myelin uptake by untreated macrophages (by Kruskal-Wallis test and Dunn's post hoc analysis).
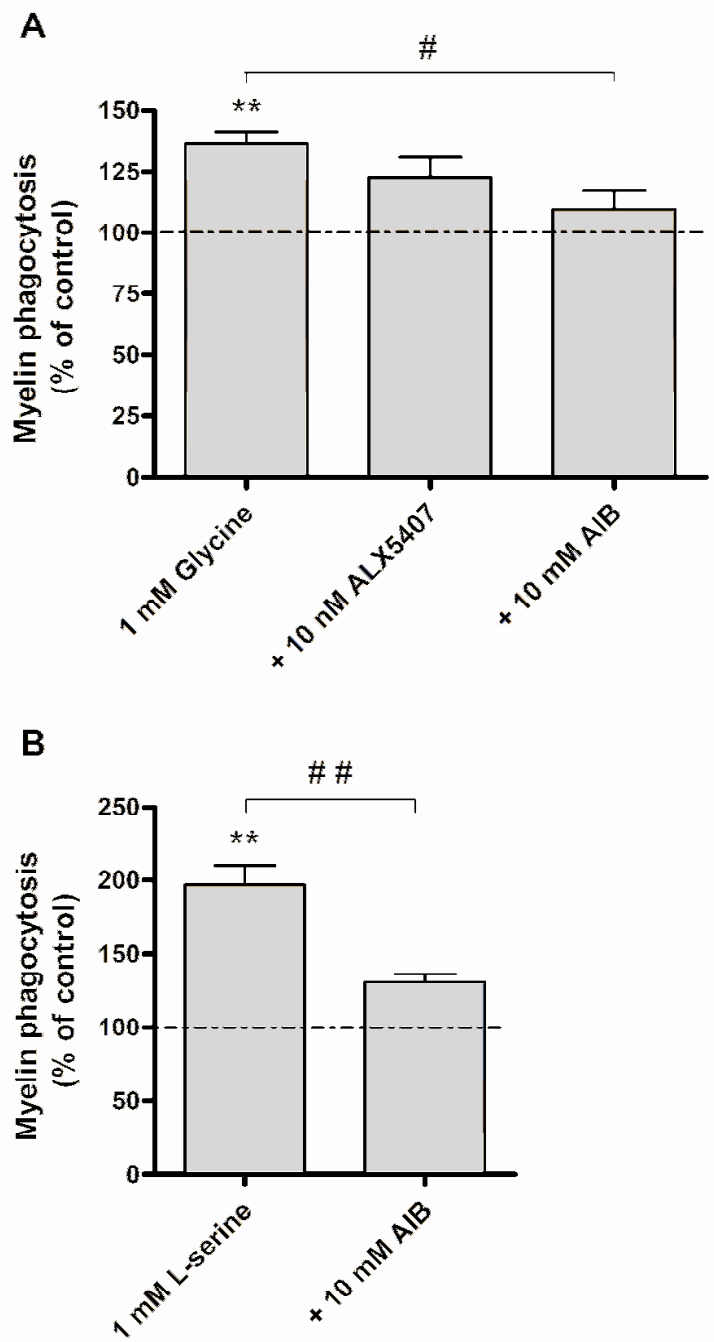

Fig. 6. Glycine modulates the myelin phagocytosis by macrophages by the activation of neutral amino acid transporters. (A) The GlyT1 inhibitor ALX5407 (10 nM) did not abolish the glycine-mediated effect on myelin phagocytosis. The NAAT substrate AIB (10 mM) partially blocked the glycine-evoked stimulation of myelin phagocytosis. (B) L-serine (1 mM) increased myelin uptake, which was again reversed by $10 \mathrm{mM}$ AIB. Myelin phagocytosis is plotted as mean percentage of myelin 
phagocytosis by untreated macrophages \pm SEM as measured by flow cytometry. Data shown are derived from at least 4 independent experiments with triplicate measurements per experiment. $\mathrm{p}<0.001$ compared to myelin uptake by untreated macrophages. \# $\mathrm{p}<0.01 ; \# \# \mathrm{p}<0.001$ compared to $1 \mathrm{mM}$ glycine/L-serine treated macrophages (by Kruskal-Wallis test and Dunn's post hoc analysis).
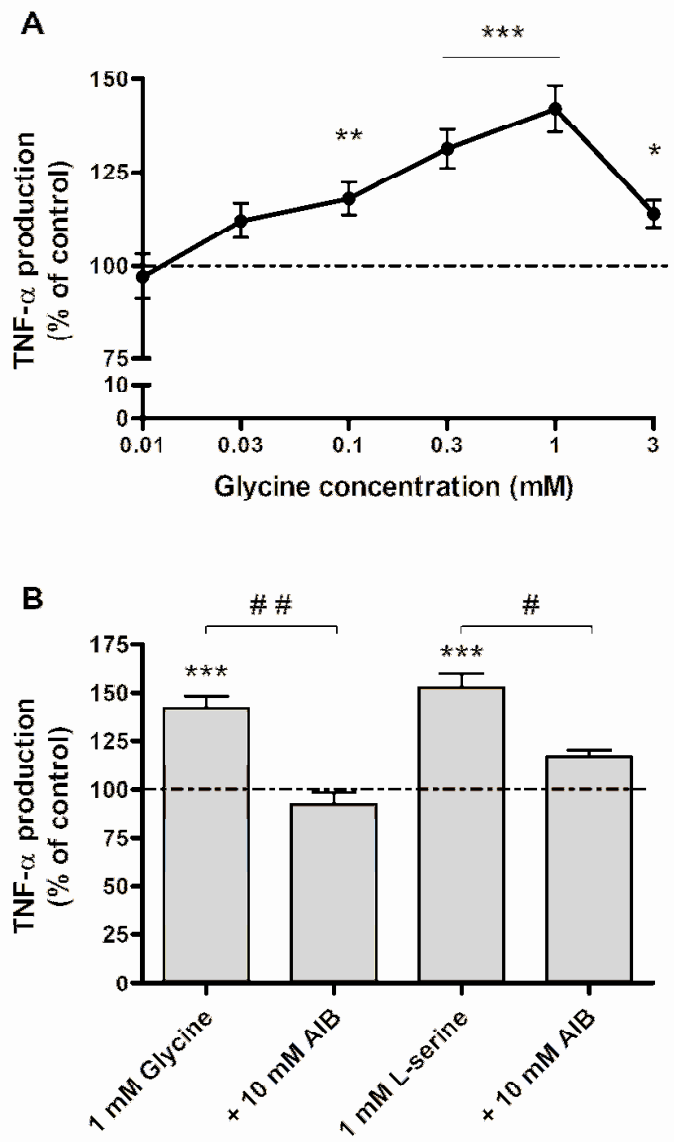

Fig. 7. Glycine increases LPS-induced TNF-a production by activation of neutral amino acid transporters. Isolated peritoneal macrophages were incubated with glycine or L-serine for $24 \mathrm{~h}$, after which $10 \mathrm{ng} / \mathrm{ml}$ LPS was added for $12 \mathrm{~h}$. (A) Glycine stimulated TNF-a secretion by macrophages in a concentration-dependent manner. (B) $10 \mathrm{mM}$ AIB counteracted the increase of TNF-a production induced by $1 \mathrm{mM}$ glycine and $1 \mathrm{mM}$ L-serine. Data shown are plotted as mean percentage of TNF-a production by LPS-stimulated untreated macrophages \pm SEM. $100 \%$ corresponds with a mean of $6.3 \pm$ $0.4 \mathrm{ng} / \mathrm{ml}$ TNF-a. Data shown are derived from at least 4 independent experiments with triplicate 
measurements in each experiment. $* \mathrm{p}<0.05 ; * * \mathrm{p}<0.01 ; * * * \mathrm{p}<0.001$ compared to TNF-a production by LPS-stimulated untreated macrophages. \# $\mathrm{p}<0.01$; \# $\mathrm{p}<0.001$ compared to $1 \mathrm{mM}$ Lserine/glycine treated LPS-stimulated macrophages (by Kruskal-Wallis test and Dunn's post hoc analysis). 